\title{
REMOTE STORAGE
}

\section{JANET, Charles}

\section{SUR LA PARTHÉNOGÉNĖSE ARRHÉNOTOQUE}

\section{DE LA FOURMI OUVRIĖRE}

Extrait des Mémoires de la Société Académique de l'Oise. - Année 1909.

\section{BEAUVAIS}

IMPRIMERIE DÉPARTEMENTALE DE L'OISE

15, rue des Flageots. 


\title{
SUR \\ LA PARTHÉNOGÉNĖSE ARRHÉNOTOQUE
}

\author{
de la Fourmi ouvrière
}

On appelle Parthénogénèse dzierzonienne ou arrhénotoque (de a,pevoroxos, qui produit des mâles) le fait, maintenant á peu près universellement admis, que, conformément aux vues de Dzierzon sur les Abeilles; les œufs de ces Insectes, ainsi que ceux des Fourmis et des Guêpes, pondus par une reine et non fécondés par un spermatczoïde, donnent exclusivement des mâles. Par contre, les femelles, c'est-à-dire les ouvrières et les reines, proviendraient probalolement toujours d'œufs fécondés.

Iì est, parait-il, d'observation courante. parmi les apiculteurs qui contrôlent méthodiquement leurs élevages et leurs croisements, que les mères fécondées par un mâle d'une autre race ne donnent que des mâles de leur propre race; tandis que les mâles issus des filles de ces mères présentent des caractères d'hybridité. C'est ce que l'on peut traduire en disant que les mâles d'Abeilles ne sont fils que de leur mère, mais sontpetits-fils à la fois d'une grand'mère et d'un grand-père, ou, en disant, plus simplement, que !es mâles d'Abeilles ont un grand père mais n'ont pas de père. Cela est bien d'accord avec la théorie de Dzierzon.

Les ouvrières sont considérées comme ne pouvant pas être fécondées et, pour ce motif, comme ne pouvant produire que des mâles.

Pour les ouvrières des Fourmis, les expériences de plusieurs auteurs (Lespès, Forel, Dewitz, Lubbock, Janet, Fielde, etc.) ont, jusqu'ici, confirmé l'exactitude d'une telle parthénogénèse.

J'ai donc été très surpris de lire dans une note de Reichen- 
bach, de Francfort-sur le Mein, l'observation suivante :*Au printemps de 1899, j'ai placé, dans un nid d'observation d'après Janet, onze ouvrières de Lasius niger L. Je les ai nourries avec du sucre inverti et des Vers de farine coupés en morceaux. Au bout de peu de jours je vis plusieurs petits tas d'œufs pondus par ces ouvrières .. A mon grand étonnement les larves [issues de ces œufs] donnèrent des ouvrières... Des ouvrières peuvent donc provenir, chez le Lasius niger, d'oeufs non fécondés, pondus par les ouvrières... Bientôt les petits tas d'œufs augmentèrent. Vers la fin de juin, le nombre des ouvrières dépassait une centaine et un grand nombre (eine Menge) de larves et de cocons élaient activement soignés. L'appétit de la colonie était excellent; les mangeoires étaient. chaque matin, complètement vidées... Dans la dernière semaine de juillet, pour ainsi dire en même temps que l'on voyait les mâles et les reines ailés de Lasius niger, des essaims nuptiaux, courir dans les jardins et dans les rues de Francfort, il y eut, dans ma colonie, éclosion d'environ une douzaine de beaux mâl's,... qui, après avoir acquis leur coloration définitive, passèrent dans la chambre éclairée du nid et y circulèrent activeinent. Si l'évasion avait été possible pour eux, ils auraient certainement pris part. en liberté, aux vols nuptiaux.

La colonie hiverna bien, et au printemps de 1900 il y eut, de bnouvcau, une rapide augmentation due aux œufs pondus par les ouvrières. Le 1er août .... le nid contenait environ $300 \mathrm{ou}$. vrières et deux ou trois douzaines de mâles. Cette année encore l'apparition des mâles coïncida avec l'époque des essaimages.

En 1901, les mêmes faits se reproduisirent, àvec celte différence. cependant, que le nombre des individus était plus faible. Cette fois encore, il y eut quelques mâles vers la fin de juillet.

Au printemps de 1902, il y avait encore environ 20 ouvrières qui élevaient des larves. mais, par suite de causes demeurées inconnues, la colonie périt vers la fin d'avril.(H. Reichenbach, Ueber Parthenogenese bei A meisen und andere Beobachtungen an Ameisenkolonien in kïnstlichen Nestern. Biol. Centralbl. T. 22, p. 461 ) 
Vu l'importance d'une telle observation, j'ai essayé, mais en vain et non sans grande dépense de temps, de la répéter. Je l'ai fait, noll pas dans un, mais dans trente nids artificiels. J'ai installé des colonies faibles et des colonies nombreuses; des colonies de vieux et des colonies de jeunes individus, et, enfin, des colınies inconteslablement vierges, formées de cocons accompagnés de 10 à 15 jeınes ouvrières écloses sous mes yeux. Jai varié le; conditions d'alimentation et celles de chaleur. Toujours, invariablement, je n'ai obtenu que des mâles.

L'année prochaine, je reprendrai ces expériences avec des ouvrières qui auront été maintenues, dans des conditions aussi variées que possible, avec des mâles.

Il serait très intéressant de voir Reichenbach recommencer ses expériences en s'entourant des précautions les plus minutieuses et, surtout, en installant, non pas un élevage isolé, mais plusieurs, afin de bien différencier les conditions daus lesquelles des faits exceptionnels pourraient se produire.

Pour des expériences de ce genre, il faut prendre des précautions d'une minutie extrême et tenir compte des causes d'erreur les plus imprévues.

Il faut, par exemple, bien veiller à ce que des Fourmis étrangères ne viennent pas s'installer dans les appareils.

Parmi les élevages que j'ai faits, l'année dernière, deux avaient été inslallés dans des conditions fournissant, chaque jour, une différence de température très considérable. Ils élaient placés sur une table, dans un grenier bas, couvert en zinc, oú la température, assez peu élevée pendant la nuit, montail considérablement les jours où le soleil frappait la toiture.

Le premier de ces élevages, installé dans un nid formé de quatre vastes chambres (trois obscures et une éclairée) contenait quatre ou cjnq mille ouvrières issues de cocons qui avaient été simplement tamisés et parmi lesquels se trouvait un très faible nombre de petits cocons mâles el, très probablement, quelques œufs et quelques jeunes larves introduits accidentellement dans les récoltes qui provenaient d'une vingtaine de nids naturels.

Le second nid était de mêmes dimensions mais contenait seulement cinquante ouvrières, de tailles variées, provenant 
de quarante cocons attentivement examinés, un à un, à la loupe, pour éviter l'apport de tout œuf el de toute larve, et de dix jeunes ouvrières écloses sous mes yeux.

Au moment de mon départ pour les vacances, les deux élevages étaient en bon état; le second contenait, encore, environ 48 ouvrières et, cians les deux, il y avait des paquets d'œufs récemment pondus.

Trois semaines plus tard, on m'écrivait que le nombre des ouvrières contenues dans le deuxième nid était de plusieurs centaines et, á mon retour, le nombre en était encore augmenté. La cause de ce qui s'était passé était, en réalité, fort simple. Lans le premier nid les habitants étaient si nombreux et les chambres obscures si encombrées qu'il y avait toujours plusieurs centaines d'individus circulant dans la chambre éclairée et, en particulier. dans l'abreuvoir et dans les mangeoires. Chaque jour, en renouvelant ces dernières, on enlevait, sans pouvoir l'éviter, au moins une trentaine de Fourmis. Les mangeoires à netloyer étaient abandonnées, avec les Fourmis qu'elles contenaient, dans une cuvette en porcelaine placée sur la même table que les élevages et, comme les Fourmis ainsi libérées disparaissaient assez rapidement et que l'on n'en voyait que cinq ou six circulant sur la table, on ne s'en occupait pas davantage.

Mais le second nid, celui, qui à l'origine, ne contenait que 50 ouvrières, se trouvait avoir été moulé avec du plâtre gâché trop clair et qui, à la prise, n'était pas devenu assez dur. Poussées par leur instinct de creuser des galeries, et profitant d'une amorce produite par une bulle d'air, les ouvrières avaient pratiqué un étroit couloir qui mettait l'intérieur de leur nid en communication avec l'extérieur.

Je constatai qu'au lieu de disparaître au loin, les Fourmis libérées par le changement journalier des mangeoires circulaient pendant quelque temps, sur la table, autour des deux nids, finissaient par trouver le petit orifice du nid en plâtre mou, y pénétraient et s'y installaient définitivement. Malgré les conditions défectueuses de ces deux élevages, je continuai á les observer et constatai que les oufs pondus pendant la belle saison donnèrent exclusivement des mâles.

Il y a quelques années un petit groupe de Lasius alienus, sans reine, est venu s'établir, spontanément, dans un de mes 
nids artificiels qui était resté abandonné, depuis l'année précédente, sur le sol, dans un angle un peu humide d'un petit hangar situé dans mon jardin. Cet appareil avait été abandonné comme défectueux, parce que son couvercle, un peu incurvé, ne le fermait pas complètement. Il en résultait un petit espace par lequel les Fourmis circulaient avec leur progéniture. Le passage libre élait long, mais n'avait pas une hauteur suffisante pour le passage d'une reine.

J'ai elı ce moment, sur une pelouse de mon jardin, un appareil, bien fermé en dessus par une vitre et une plaque de plâtre, pourvu en dessous d'un petit orifice et qui est utilisé par des Formıca fusca. J'avais placé cet appareil, rempli de terre, sur un petit nid naturel de Tetramorium et je pensais que la colonie y étendrait son nid comme je l'ai obtenu déjá plusieurs fois. L'année suivante, il n'y avait plus de Tetamorium, mais une belle colonie de Formica fusca s'était installée dans la terre au-dessous de l'appareil. Elle avait, presque complètement, vidé les chambres de la terre fine qui les remplissait et elle les utilisait, les jours de beau temps, comme chambres de chauffe pour le couvain.

Relalivement á l'apparition des mâles aı moment voulu pour les essaimages nuptiaux, je ferai remarquer qu'il n'a pas été fait, jusqu'ici, d'observation précise démontrant qu'une reine de Fourmi ait, chaque année une période délerminée de ponte d'œufs mâles.

Chez les Abeilles, la ponte d'œufs mâles est déterminée par une contraction ou par l'absence d'une contraction musculaire; ce fait est déterminé par la rencontre d'un alvéole de dimensions spéciales; la construction de cet alvéole par les ouvrières est déterminée, dans tout jeune essaim, à un certain moment, par l'apparition de causes, sans doute assez précises, mais non encore bien mises en évidence.

Il n'est pas impossible qu'il y ait aussi, chez chaque reine de Fourini, au cours de sa période d'existence imaginale reproductrice, période qui peut durer plus de dix années, une intercalation annuelle d'une période de ponte mâle déterminée, au moment utile, par des causes non précisées. Toutefois, non seulement je ne suis pas encore parvenu á le démontrer, mais il y a, au contraire, certains faits qui sembleraient 
indiquer que cette période pourrait bien ne pas exister. Dans ce cas, en dehors d'un petit nombre de mâles provenant d'œufs qui auraient accidentellement échappé à la fécondation (c'est sans doute le cas d'un mâle que j'ai trouvé isolé, au milieu du premier gâteau d'un nid de Vespa crabro, gâteau qui ne contenait que des ouvrières) tous les mâles des vols nuptiaux d'une espèce de Fourmis proviendraient uniquement de la ponte de reines non fécondées ou ayant épuisé leur approvisionnement de spermatozoïdes, et de la ponte des ouvrières des colonies orphelines ou des colonies exceptionnellement bien approvisionnées en nourriture.

S'il y avait une intercalation normale, annuelle, d'une période de ponte mâle, on trouverait, simultanément, dans chacun des nids d'une même espèce une certaine proportion, assez régulière, entre le nombre des mâles et celui des jeunes reines. Or, si avant l'époque des vols nuptiaux on examine un grand nombre de nids, on coristate qu'il y en a toujours beaucoup oú les individus ailés, à l'état de nymphe ou à l'état d'imago, sont surtout, ou même exclusivement soit mâles, soit reines.

A l'appui de l'hypothèse de l'absence d'une intercalation annuelle, régulière et normale, d'une période de ponte mâle chez les reines de Fourmis, je rappelle qu'une reine qui a été conservée par Lubbock pendant piusieurs années n'a donné abșolument que des ouvrières, et que j'ai fait moimême, exactement la même observation, sur une reine de Lasius alienus qui, après avoir, une deuxième fois, fondé une nouvelle colonie, n'a, durant les neuf années pendant lesquelles elle est restée en observation dans mon laboratoire, donné absolument que des ouvrières, sans intercalation d'apparition d'un seul mâle.

Relalivement à la fécondation ou à la non fécondation d'un œuf produit par une reine de Fourmi, de Guêpe ou d'Abeille, on admet, en général, que la fécondation est empêchée par une occlusion momentanée de l'orifice du réceptacle séminal tandis que la fécondation est produite par une concontraction de ce réceptacle. Quelques observations encore incomplètes, qui n'ont porté que sur les Fourmis, m'ont con- 
duit à admettre un processus tout à fait différent de ceux indiqués ci-dessus.

Voici, pour moi, comment les choses se passent probablement. Les spermatozoïdes agglutinés par le liquide fourni par la glande du réceptacle séminal, forment dans ce réceptacle, une masse de réserve et, dans son canal, un cordon fécondateur. Dans cette masse et dans ce cordon, les spermatozoïdes demeurent immobiles, dans un état de vie latente qui peut durer une dizaine d'années. Les spermatozoïdes ne retrouvent leur activité qu'au moment où, par suite de la disparition du liquide agglutinateur, ils sont mis à découvert à l'extrémité libre du cordon fécondateur, extrémité qui se trouve juste à l'orifice du canal du réceptacle.

Lorsque l'œuf descend au travers de l'utérus, sans subir aucun arrêt, il passe devant l'orifice du canal du réceptacle séminal sans arriver au contact de cet orifice.

Il n'en est plus de même si, au moment où l'œuf est sur le point de sortir de l'utérus, ce dernier se contracte. Dans ce cas, l'œuf est refoulé un peu obliquement vers le haut; il se trouve très étroitement coilfé par le cul-de-sac sur lequel s'ouvre le canal du réceptacle, et l'un des spermalozoïdes actifs de l'extrémité du cordon fécondateur, pénètre dans le micropyle, tandis que les autres spermatozoïdes, non encore suffisamment dégagés, sont retenus dans ce cordon.

La fécondation ou la non fécondation résulterait ainsi, uniquement, de la contraction ou de la non contraction des fibres musculaires de l'extrémité distale de l'utérus.

Pour mieux saisir les explications ci-dessus, on pourra se reporter á la planche 1 de mon travail : "Anatomie du Gaster de la Myrmica rubra » oú le réceptacle séminal, sa glande et son canal á extrémité incurvée, sont figurés dans la position qu'ils occupent au repos.

Imprimerie départementale de l’Oıse, 15, rue des Flageots, Beauvais. 